\title{
ESTIMACIÓN DE LOS EFECTOS DE TORSIÓN ACCIDENTAL SÍSMICA A PARTIR DE LA AMPLIFICACIÓN DE LA RESPUESTA DE UN MODELO TORSIONALMENTE BALANCEADO
}

\author{
Gustavo Santos Gálvez Cortés ${ }^{(1)}$, Jaime De la Colina Martínez ${ }^{(2)}$ y Jesús Valdés González ${ }^{(2)}$
}

\begin{abstract}
RESUMEN
Mediante simulación Monte Carlo y el programa de análisis Canny-E, se analizan dinámicamente ocho modelos estructurales elásticos representativos de edificios de marcos de concreto reforzado de 4, 7 y 10 niveles, con el objetivo de establecer un procedimiento simplificado para tomar en cuenta los efectos de la torsión accidental en el diseño sísmico de edificios. Los resultados muestran que es factible diseñar por torsión sísmica un edificio a base de marcos, representativo de edificios de oficinas, empleando una relación simple que amplifica la respuesta del correspondiente modelo torsionalmente balanceado para estimar los elementos mecánicos y cinemáticos de diseño.
\end{abstract}

Palabras clave: excentricidad accidental; simulación Monte Carlo; análisis dinámico; diseño sísmico; torsión sísmica

\section{ESTIMATION OF THE SEISMIC ACCIDENTAL-TORSION EFFECTS FROM THE AMPLIFICATION OF THE RESPONSE OF A TORSIONALLY BALANCED MODEL}

\begin{abstract}
Eight structural models representing 4, 7 and 10-level reinforced concrete frames are dynamically analyzed via Monte Carlo simulation and the Canny-E analysis program. The aim is to establish a simplified procedure to take into account the effects of the accidental torsion in the seismic design of buildings. The results show that it is possible to design a typical office frame building to withstand the accidental-torsion effects by using a simple relationship that amplifies the response of the corresponding torsionally balanced model to estimate the design forces and displacements.
\end{abstract}

Keywords: accidental torsion; accidental eccentricity; seismic design; seismic torsion; Monte Carlo simulation

\footnotetext{
Artículo recibido el 30 de noviembre de 2017 y aprobado para su publicación el 12 de abril de 2019. Se aceptarán comentarios y/o discusiones hasta cinco meses después de su publicación

(1) Estudiante de maestría. Facultad de Ingeniería, Universidad Autónoma del Estado de México, Cerro de Coatepec S/N, Ciudad Universitaria, Toluca, México C.P. 50100.gusantos.gc@gmail.com

(2) Profesor - Investigador. Facultad de Ingeniería, Universidad Autónoma del Estado de México, Cerro de Coatepec S/N, Ciudad Universitaria, Toluca, México C.P. 50100.jaime_delacolina@yahoo.com; jvaldes@uaemex.mx
} 


\section{INTRODUCCIÓN}

El diseño sísmico de edificios considera que las estructuras pueden girar alrededor de un eje vertical. Este comportamiento se conoce como torsión sísmica. Los reglamentos de diseño por sismo incluyen el efecto de la torsión sísmica a través de un momento de torsión. El cálculo de dicho momento recurre al concepto de excentricidad de diseño, la cual se supone como la suma de dos excentricidades: la natural $\left(e_{s}\right)$ y la accidental $\left(e_{a}\right)$.

La excentricidad natural se puede estimar a nivel de proyecto a partir de los valores nominales de las rigideces de los elementos estructurales y de la distribución supuesta de masas en el modelo. La excentricidad accidental se asume como una distancia a incluir en la excentricidad de diseño, por las posibles variaciones en rigideces y masas en el momento del sismo. Los reglamentos de diseño prescriben que la excentricidad accidental se estime como un porcentaje $(\beta)$ de la dimensión en planta del edificio $(b)$ perpendicular a la dirección del cortante sísmico. El reglamento International Building Code (ICC, 2009), (ASCE, 2010) y el código europeo (ECS, 2004) estipulan la excentricidad accidental como $0.05 b$. Por su parte, el reglamento de la ciudad de México (RC CdMx-2017), especifica un valor que disminuye linealmente desde $0.10 b$ para el entrepiso superior del edificio hasta $0.05 b$ para el inferior. Esta aproximación toma en cuenta algunos resultados que revelan que la torsión accidental disminuye hacia la base del edificio (De-la-Colina, et al., 2011). La falta de consenso entre los reglamentos de diseño para estimar la excentricidad accidental de proyecto sugiere que las cláusulas ofrecidas requieren mayor estudio.

Las excentricidades accidentales de edificios son sólo valores útiles para diseño (García, 2015). En realidad, la excentricidad accidental es de naturaleza aleatoria, por lo que el valor estipulado por los reglamentos es solamente una aproximación determinista. Estudios recientes que han abordado el problema de la torsión accidental (Nelson, 2001; Escobar et al., 2002; Hernández and López, 2004; Escobar et al., 2004; De la Colina et al., 2011; García, 2015; De la Colina et al., 2016) sugieren proponer otros procedimientos para tomar en cuenta sus efectos en el diseño sísmico de edificios.

Por otro lado, algunos autores (Padilla, 2002; Escobar et al., 2004) mencionan que el uso de las recomendaciones de los reglamentos en modelos tridimensionales de estructuras conduce en sentido estricto a realizar un número de análisis igual a $4^{n p}$ donde $n p$ es el número de pisos.

Estudios previos (De-la-Llera y Chopra 1994) muestran que los efectos de la torsión accidental son mayores en edificios torsionalmente balanceados que en edificios desbalanceados. En este estudio se analizaron edificios con $e_{s}=0$, con el propósito de maximizar el efecto de la torsión accidental. El componente rotacional del movimiento del terreno es de baja magnitud, por lo cual se desprecia su contribución a los efectos de la torsión accidental en este trabajo.

A partir de los argumentos anteriores, en este trabajo se propone un procedimiento simplificado que requiere un sólo análisis para incorporar los efectos de la torsión accidental en el diseño sísmico de edificios. El procedimiento usa una relación simple empleando un factor que amplifica la respuesta del correspondiente modelo torsionalmente balanceado para estimar los valores de diseño. Este procedimiento puede emplearse como un método simple para tomar en cuenta la torsión accidental en el diseño sísmico de edificios regulares a base de marcos. El procedimiento se enfoca a edificios con distribuciones uniformes de masa y rigidez, tanto en planta como en elevación. Asimismo, se anticipa que su aplicación debiera limitarse a edificios que por lo menos tengan tres marcos en cada dirección ortogonal. 


\section{PROCEDIMIENTO SIMPLIFICADO PARA INCLUIR LA TORSIÓN ACCIDENTAL EN EL DISEÑO SÍSMICO DE EDIFICIOS}

El procedimiento propuesto tiene como objetivo principal, presentar un método simple para estimar los elementos mecánicos y cinemáticos de diseño debidos a la torsión accidental, en edificios de marcos para oficinas. El procedimiento utiliza la siguiente relación (ecuación 1) en la cual la estimación del parámetro de diseño que se calcula tiene aproximadamente una probabilidad de $2 \%(p \approx 0.02)$ de ser excedida:

$$
E_{\text {dea2 }}=A_{e a 2} * E_{\text {bal }}
$$

donde:

$E_{b a l}$ : elemento mecánico o cinemático de cualquier elemento estructural que conforme el edificio. Se obtiene de un único análisis dinámico lineal del correspondiente modelo torsionalmente balanceado del edificio que desea diseñarse. Así, $E_{b a l}$ puede representar momentos flexionantes, fuerzas cortantes, esfuerzos normales, etc. Se aclara que la condición torsionalmente balanceada implica que $e_{s}=0$ y $e_{a}=0$.

$E_{\text {dea2 }}$ : elementos mecánicos o cinemáticos de diseño por sismo correspondientes a $E_{\text {bal }}$ pero tomando en cuenta los efectos de la torsión accidental. Esta estimación tiene una probabilidad $p=0.02$ de ser excedida.

$A_{e a 2}:$ factor de amplificación por torsión accidental aplicable a todos los elementos mecánicos o cinemáticos de diseño de cualquier elemento estructural. Este factor tiene un promedio igual a 1.20. La justificación del valor de este factor se presenta más adelante.

Los pasos del procedimiento simplificado de diseño por sismo propuesto se describen a continuación:

1. Obtener $E_{b a l}$ realizando un análisis dinámico lineal del edificio a diseñarse torsionalmente balanceado.

2. Obtener $\operatorname{los} E_{\text {dea2 }}$ amplificando las respuestas anteriores por $A_{e a 2}=1.20$.

\section{METODOLOGÍA PARA EL CÁLCULO DE FACTORES $A_{e a}$ CON $P=0.02$}

El factor de amplificación $A_{e a 2}=1.20$ recomendado en el procedimiento propuesto se obtuvo del análisis estadístico de factores $A_{e a}$ con $p=0.02$. En esta sección se describe el procedimiento seguido para la obtención de dichos factores.

Se utilizó el Método de Monte Carlo (MMC) para simular los efectos de la torsión accidental en un total de ocho modelos representativos de edificios de concreto reforzado con uso de oficinas de 4, 7 y 10 niveles sometidos a un registro sísmico en dos direcciones ortogonales.

Para cada modelo se realizaron 10,000 análisis lineales paso a paso. Se obtuvo la misma cantidad de observaciones de cada parámetro de respuesta seleccionado en esta investigación, dichos parámetros se presentan en detalle más adelante. Las 10,000 observaciones obtenidas para un parámetro de respuesta se denominaron $\overline{E_{d e a}}$. Se utiliza la notación con línea sobre las variables para denotar vectores. 
Una vez obtenidas las 10,000 observaciones de cada modelo y cada parámetro de respuesta de cada elemento estructural, se normalizaron los 10,000 datos contenidos en $\overline{E_{\text {dea }}}$ respecto a los valores obtenidos al realizar un análisis lineal paso a paso considerando $e_{s}=0$ y $e_{a}=0$.

Los resultados del análisis mencionado se identifican de manera general como $E_{b a l}$. La normalización da lugar a los denominados factores de amplificación por excentricidad accidental $\left(\overline{A_{e a}}\right)$. Dichos factores se trataron estadísticamente para obtener histogramas de $A_{e a}$, un histograma para cada parámetro de respuesta y para cada elemento estructural.

Finalmente, de cada histograma se obtuvo un factor $A_{e a}$ con $p=0.02$. El diagrama de la figura 1 resume las actividades realizadas para calcular los factores $A_{e a}$ y $A_{e a} \operatorname{con} p=0.02$.

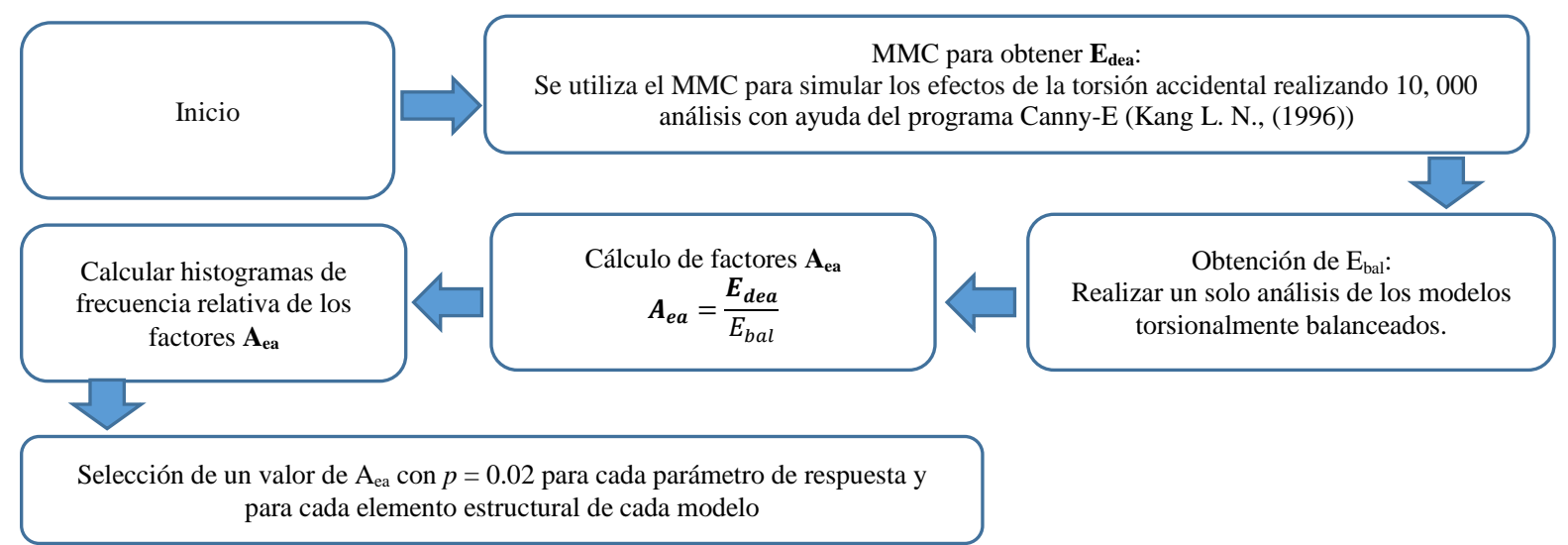

Figura 1. Diagrama de flujo para el cálculo de factores $A_{e a}$ con $p=0.02$ para cada modelo y para cada parámetro de respuesta

\section{MODELOS ESTRUCTURALES Y REGISTRO SÍSMICO}

Las geometrías de los modelos se limitan a estructuras regulares en planta seleccionadas para estudiar los efectos de la torsión accidental en modelos con plantas cuadrada y rectangular. En cada modelo, se consideró dos relaciones de aspecto en planta: 1:1 y 1:2. Se usan modelos con 4, 7 y 10 niveles. Las alturas de entrepiso se consideraron de $3.0 \mathrm{~m}$. En todos los casos se supone que la losa de cada piso es un diafragma rígido en su plano.

En caso de tener edificios de mayor altura el sistema estructural requerido probablemente sería distinto al sistema de marcos considerado en este trabajo. Los modelos analizados en este estudio no necesariamente representan edificios reales. Aquí sólo se busca que los modelos tengan las propiedades dinámicas generales de edificios a base de marcos de concreto reforzado. Todos los modelos usados en este estudio son simétricos respecto a los planos verticales que contienen los ejes principales de la planta.

En resumen, se tienen 8 modelos estructurales representativos de edificios de marcos de concreto reforzado para uso de oficinas: 4 modelos con crujías de $6.0 \mathrm{~m}$ en dirección $X$ y dirección $Y$ para 4 y 7 niveles; 4 modelos con crujías de 8.0 m en dirección $X$ y dirección $Y$ para 7 y 10 niveles.

Las masas de los modelos provienen de la carga muerta y de la carga viva instantánea. La primera es considerada como determinista (tabla 1); mientras que la segunda se supone como variable aleatoria. Las 
secciones trasversales de columnas y trabes, así como los valores de la carga muerta para los ocho modelos se resumen en la tabla 1.

La tabla 1 contiene una identificación abreviada de cada uno de los modelos estudiados. Por ejemplo, en la etiqueta del modelo M4C6, el primer número se refiere al número de pisos, la segunda letra se refiere a la relación en planta de cada modelo (cuadrado o rectangular) y el número final indica las dimensiones de las crujías en metros.

Tabla 1. Modelos, dimensiones nominales de trabes y columnas, y cargas muertas

\begin{tabular}{|c|c|c|c|c|c|c|}
\hline Modelo & $\begin{array}{l}\text { Dimensión en planta } \\
\text { y tamaño de crujías (m) }\end{array}$ & $\begin{array}{l}\text { Sección } \\
\text { de columnas }(\mathrm{cm})\end{array}$ & transv & rsal & $\begin{array}{l}\text { Sección transversal } \\
\text { de trabes }(\mathrm{cm})\end{array}$ & $\begin{array}{l}\text { Carga muerta } \\
\left(\mathrm{kg} / \mathrm{m}^{2}\right)\end{array}$ \\
\hline M4C6 & $\begin{array}{l}\text { En dirección X: } 18.00 \\
\text { En dirección Y: } 18.00 \\
\text { Crujías a cada: } 6.00 \\
\text { En dirección X: } 24.00 \\
\text { En dirección Y: } 12.00 \\
\text { Crujías a cada: } 6.00\end{array}$ & $\begin{array}{lcc}\text { Pisos } & 1-2: & 50 \\
\text { Pisos } 3-4: & 40 \times & 40\end{array}$ & $\mathrm{x}$ & 50 & $\begin{array}{l}\text { Todos los pisos: } \\
50 \times 25\end{array}$ & $\begin{array}{c}\text { Todos los pisos: } \\
450.00\end{array}$ \\
\hline $\begin{array}{l}\text { M7C6 } \\
\text { M7R6 }\end{array}$ & $\begin{array}{l}\text { En dirección X: } 18.00 \\
\text { En dirección Y: } 18.00 \\
\text { Crujías a cada: } 6.00 \\
\text { En dirección X: } 24.00 \\
\text { En dirección Y: } 12.00 \\
\text { Crujías a cada: } 6.00\end{array}$ & $\begin{array}{l}\text { Pisos 1-4: } \quad 60 \\
\text { Pisos 5-7: } 50 \times 50\end{array}$ & $\mathrm{x}$ & 60 & $\begin{array}{l}\text { Todos los pisos: } \\
50 \times 25\end{array}$ & $\begin{array}{c}\text { Todos los pisos: } \\
450.00\end{array}$ \\
\hline $\begin{array}{l}\text { M7C8 } \\
\text { M7R8 }\end{array}$ & $\begin{array}{l}\text { En dirección X: } 24.00 \\
\text { En dirección Y: } 24.00 \\
\text { Crujías a cada: } 8.00 \\
\text { En dirección X: } 32.00 \\
\text { En dirección Y: } 16.00 \\
\text { Crujías a cada: } 8.00\end{array}$ & $\begin{array}{l}\text { Pisos 1-4: } \quad 60 \\
\text { Pisos 5-7: } 50 \times 50\end{array}$ & $x$ & 60 & $\begin{array}{l}\text { Todos los pisos: } \\
\quad 60 \times 25\end{array}$ & $\begin{array}{c}\text { Todos los pisos: } \\
550.00\end{array}$ \\
\hline M10C8 & $\begin{array}{l}\text { En dirección X: } 24.00 \\
\text { En dirección Y: } 24.00 \\
\text { Crujías a cada: } 8.00 \\
\text { En dirección X: } 32.00 \\
\text { En dirección Y: } 16.00 \\
\text { Crujías a cada: } 8.00\end{array}$ & $\begin{array}{lrr}\text { Pisos 1-4: } & 70 \\
\text { Pisos } & 5-7: & 60 \\
\text { Pisos 8-10: } & 50 \times 50\end{array}$ & $\begin{array}{l}\mathrm{x} \\
\mathrm{x}\end{array}$ & $\begin{array}{l}70 \\
60\end{array}$ & $\begin{array}{l}\text { Todos los pisos: } \\
\quad 60 \times 25\end{array}$ & $\begin{array}{c}\text { Todos los pisos: } \\
550.00\end{array}$ \\
\hline
\end{tabular}

\section{Materiales de los elementos estructurales}

Los modelos se conforman de marcos de concreto reforzado con $\mathrm{f}^{\prime} \mathrm{c}=300 \mathrm{~kg} / \mathrm{cm} 2$ y un módulo de elasticidad $E=242,487 \mathrm{~kg} / \mathrm{cm}^{2}$.

\section{Registro sísmico}

Los modelos estructurales se sometieron simultáneamente a ambos registros horizontales del sismo del 18 de mayo de 1940 ocurrido en Imperial Valley, California (El Centro). Cada componente sísmica se aplicó a lo largo de las direcciones principales de la planta de la estructura.

En principio, interesa conocer las estadísticas de los parámetros de respuesta de los casos con torsión accidental, normalizados respecto a los modelos sin torsión accidental por lo que cualquier otro registro podría utilizarse. Este estudio se limita al uso de un solo registro sísmico como una primera aproximación 
para el estudio del problema de los efectos de la torsión accidental. Cabe mencionar que dicho registro corresponde a terreno firme.

En la figura 2 se muestran los periodos a torsión de los modelos, así como los espectros de respuesta de aceleraciones de ambas componentes sísmicas. Estos espectros corresponden a un amortiguamiento del $5 \%$ del crítico.

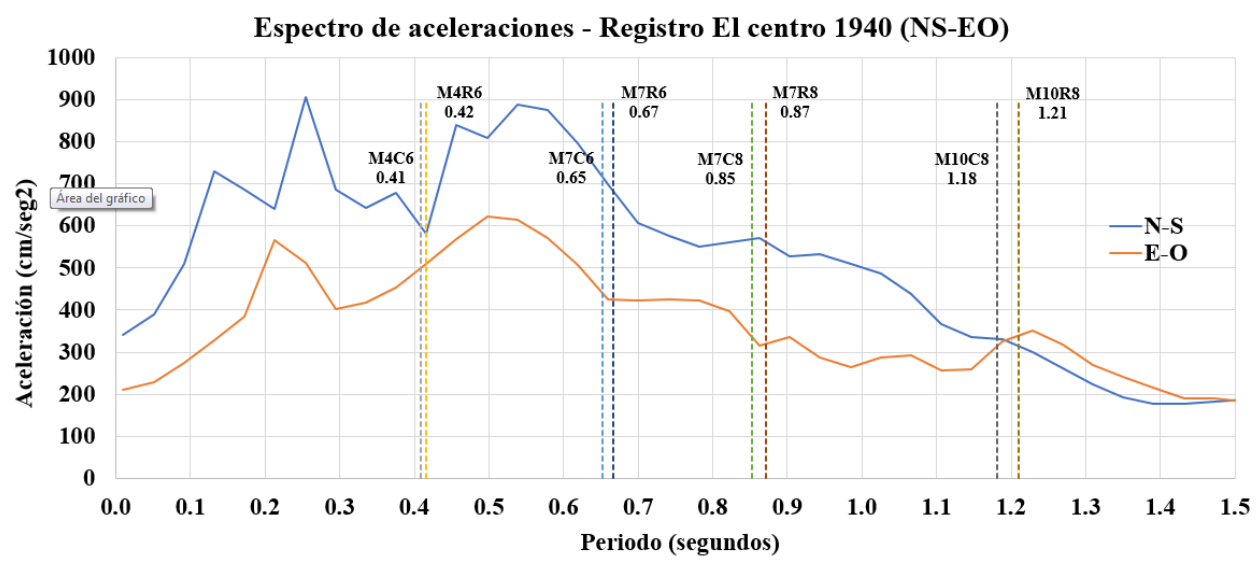

Figura 2. Espectro de aceleraciones de El Centro (NS-EO) y periodos a torsión de los modelos estudiados

\section{APLICACIÓN DEL MÉTODO DE MONTE CARLO}

Se analizaron dinámicamente ocho modelos estructurales elásticos para obtener los elementos mecánicos y cinemáticos que resultan en el caso de que un edificio presente torsión accidental durante un evento sísmico. Se utilizó el MMC para simular los efectos de la torsión accidental a partir de las variables aleatorias que la definen y obtener información de los parámetros de respuesta seleccionados descritos más adelante.

El Método Monte Carlo se aplicó de la siguiente manera:

1. Generación de los valores de las variables aleatorias utilizando sus funciones de densidad de probabilidad (fdp).

2. Aplicación de los valores generados a los modelos estructurales.

3. Cálculo de las respuestas que proveen información del comportamiento de los modelos. En cada caso, la respuesta se observa a través de los parámetros de respuesta seleccionados (tablas 3, 4 y 5).

4. Repetición de los pasos 1,2 y 3 para $N=10,000$ realizaciones, para tener la misma cantidad de observaciones de cada parámetro de respuesta.

5. Obtención de los histogramas de frecuencia relativa de los distintos parámetros de respuesta para todos los elementos estructurales de cada modelo, para examinar su comportamiento estadístico.

\section{Variables aleatorias y sus parámetros}

Las variables aleatorias consideradas en este trabajo para simular los efectos de la torsión accidental en los modelos son la magnitud y posición de la carga viva durante la ocurrencia de un sismo (carga viva instantánea), así como también las rigideces a flexión de trabes y columnas. 
Cada variable aleatoria, se define a partir de su función de densidad de probabilidad (fdp). Para los parámetros de rigidez de los elementos estructurales $\left(E I \_t, E I \_c\right)$ y la posición de la carga viva $(G X, G Y)$ se consideraron las referencias (Soriano y Ruiz, 2001), (De la Colina, et al., 2011; García, 2015). Para el valor de la media de la carga viva instantánea ( $W C V i$ ) se siguieron las recomendaciones de la Norma ASCE (ASCE, 2010) que indica que en las combinaciones de carga que incluyen cargas muertas, cargas vivas y sismo se utilice un factor de 0.5 para reducir la intensidad de la carga viva. En tal caso, la carga viva resulta en $122.01 \mathrm{~kg} / \mathrm{m}^{2}$ con un coeficiente de variación igual a 0.292 obtenido de las referencias (García, 2015) y (De la Colina, et al., 2016). La tabla 2 resume las variables aleatorias y los parámetros requeridos para definir sus respectivas fdp's.

Tabla 2. Variables aleatorias y sus parámetros

\begin{tabular}{clcccc}
\hline Abreviación & \multicolumn{1}{c}{ Variables aleatorias } & fdp & Media & $\begin{array}{c}\text { Desviación } \\
\text { estándar }\end{array}$ & $\begin{array}{c}\text { Coef. de } \\
\text { variación }\end{array}$ \\
\hline$W C V i$ & Magnitud de la carga viva & Gamma & $122.01\left(\mathrm{~kg} / \mathrm{m}^{2}\right)$ & $35.62\left(\mathrm{~kg} / \mathrm{m}^{2}\right)$ & 0.292 \\
$G X, G Y$ & Posición de la carga viva & Normal & $0.498 b(\mathrm{~m})$ & $0.037 b(\mathrm{~m})$ & 0.074 \\
$E I \_t$ & Rigidez de trabes & Normal & $E I_{\text {nominal }}$ & $0.11 E I_{\text {nominal }}$ & 0.110 \\
$E I \_c$ & Rigidez de columnas & Normal & $E I_{\text {nominal }}$ & $0.11 E I_{\text {nominal }}$ & 0.110 \\
\hline
\end{tabular}

\section{Justificación del número de simulaciones $N$}

Los trabajos de Díaz (1995), García (2015) y De la Colina, et al., (2016) han aportado criterios para la selección del número de simulaciones $(N)$ en estudios relacionados con el MMC. Un primer criterio es la estabilidad de la media y de la desviación estándar de los parámetros de respuesta. Otro criterio se basa en las recomendaciones de (Hogg y Craig, 1995) para calcular un intervalo de confianza para estimar una determinada probabilidad $p$. La proporción $p$ corresponde a la probabilidad de excedencia de los parámetros de respuesta. Utilizando el criterio anterior, para un intervalo de confianza del 95\%, $p=0.02$ y considerando un error del $10 \%$ de $p, N$ resulta igual a 9,604.

Mediante el criterio de la estabilidad de la media y de la desviación estándar, se calculó el valor promedio y la desviación estándar del momento flexionante en su extremo derecho (Mder), para todas las trabes del primer nivel del modelo M4C6. Estos resultados se grafican en la figura 3 contra el número de simulaciones $N$. Se observa que $N \approx 10,000$ permite la estabilización de los parámetros anteriores.


Figura 3. Efecto del número de simulaciones o realizaciones $(N)$ sobre la media y desviación estándar en el parámetro Mder de trabes del 1er nivel del modelo M4C6 
Dada la naturaleza del MMC un mayor número de simulaciones conduce a mayor precisión en los resultados, por lo que $N$ se seleccionó igual a 10,000.

\section{Parámetros de respuesta seleccionados}

Para determinar el factor de amplificación a usar en el procedimiento de diseño propuesto en este trabajo, se seleccionaron los parámetros de respuesta listados en las tablas 3, 4 y 5. La selección de los parámetros para trabes y columnas (ver tablas 3 y 4) obedece a que éstos proporcionan la información necesaria para su diseño. Nótese que estos parámetros se calculan para cada instante y al final del análisis se seleccionan los valores máximos.

Tabla 3. Parámetros de respuesta de trabes

\begin{tabular}{lc}
\hline Parámetros de respuesta de trabes & Abreviación \\
\hline Momento máximo flexionante en el extremo derecho de la trabe & Mder \\
Momento máximo flexionante en el extremo izquierdo de la trabe & Mizq \\
Cortante máximo de la trabe (en cualquier extremo donde se presente) & VT \\
\hline
\end{tabular}

Tabla 4. Parámetros de respuesta de columnas

\begin{tabular}{lc}
\hline Parámetros de respuesta de columnas & Abreviación \\
\hline Esfuerzo normal máximo & Esf \\
Cortante máximo en dirección X & VXC \\
Cortante máximo en dirección Y & VYC \\
\hline
\end{tabular}

Tabla 5. Parámetros de respuesta cinemáticos

\begin{tabular}{lc}
\hline Parámetro de respuesta cinemáticos & Abreviación \\
\hline Desplazamiento relativo de entrepiso máximo en la dirección X & Idmx \\
Desplazamiento relativo de entrepiso máximo en la dirección Y & Idmy \\
\hline
\end{tabular}

\section{RESULTADOS Y DISCUSIÓN}

En resumen, se aplicó el Método Monte Carlo a ocho modelos estructurales para estudiar los efectos de la torsión accidental en edificios de concreto reforzado a base de marcos, para oficinas. Se realizaron 10,000 análisis dinámicos lineales en modelos elásticos con torsión accidental simulada. Los resultados se normalizaron respecto a la respuesta obtenida de un análisis lineal de los mismos modelos, pero torsionalmente balanceados. Del análisis de estos cocientes se seleccionó un valor que puede ser empleado como factor de amplificación para el diseño por torsión sísmica.

\section{Resultados de histogramas de factores $A_{e a}$ para el modelo M4C6}

En esta sección se muestran los resultados obtenidos del modelo M4C6. La figura 4 muestra este modelo en tres dimensiones y su marco Y1. Cada marco tiene doce trabes y dieciséis columnas. Se utiliza la misma configuración del marco Y1 para mostrar los resultados de los factores $A_{e a}$ para cada parámetro de respuesta en cada elemento estructural. Aun cuando se obtienen histogramas de factores $A_{e a}$ para cada parámetro de respuesta, se utilizan gráficas de línea para mostrar los resultados de varios parámetros de 
respuesta de un solo elemento estructural. Sin embargo, la palabra histograma se mantiene en las figuras siguientes para recordar la naturaleza de los datos obtenidos.


Figura 4. Modelo M4C6 en tres dimensiones y su marco Y1


Figura 5. Histogramas de factores $A_{e a}$ en trabes - Marco X1 del modelo M4C6

Las figuras 5 y 6 muestran los histogramas de frecuencia relativa de los factores $A_{e a}$ para los tres parámetros de respuesta Mder, Mizq y VT de las doce trabes de los marcos X1 y Y1, respectivamente. Por claridad se grafican únicamente los valores de cada barra de cada histograma. El valor de los factores $A_{e a}$ se identifica en el eje horizontal mientras que las frecuencias relativas en porcentaje en el eje vertical. Los valores máximos de $A_{e a}$ para el marco X1 son del orden de 1.40, mientras que para el marco Y1 el valor máximo es de 1.60 .

En la figura 6 se observa que la cola derecha de los histogramas de los pisos superiores es más alargada que en los pisos inferiores, indicando que para un valor fijo de $A_{e a}$ la probabilidad de exceder dicho valor es mayor en los pisos superiores que en los inferiores. Este resultado coincide con los de otros autores (De la Colina, et al., 2011; García, 2015 y De la Colina, et al., 2016), en el sentido de que, en un edificio, la torsión accidental es mayor para los entrepisos superiores que para los inferiores. 

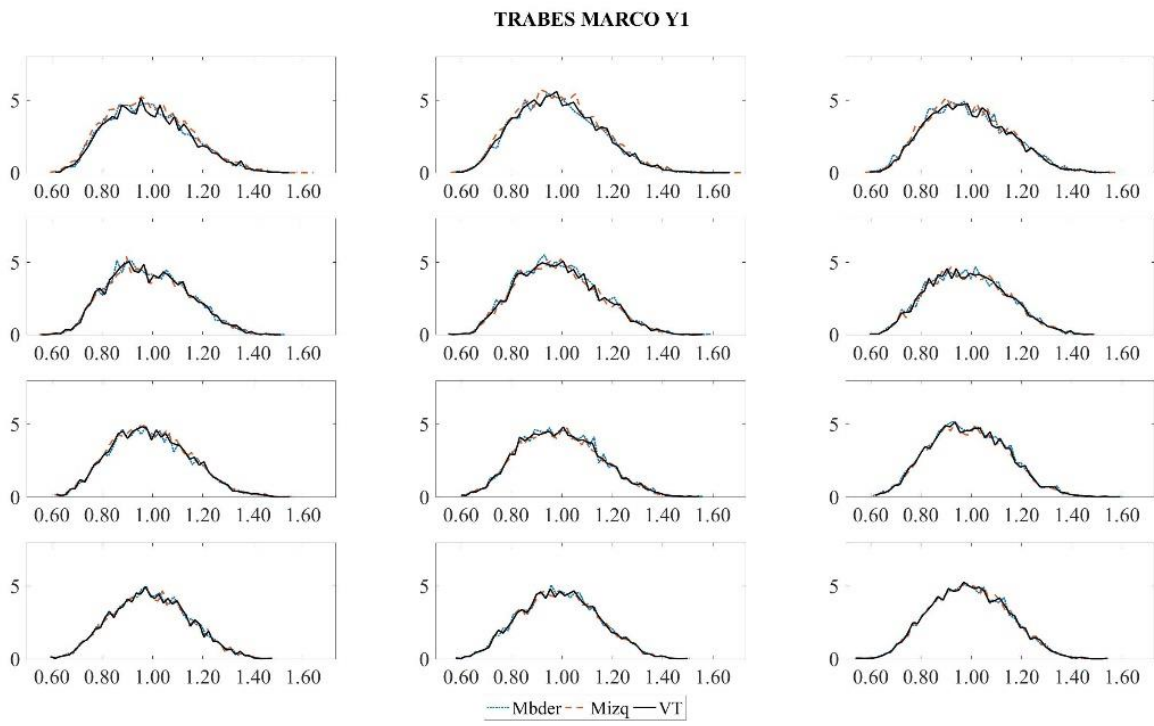

Figura 6. Histogramas de factores $A_{e a}$ en trabes - Marco Y1 del modelo M4C6
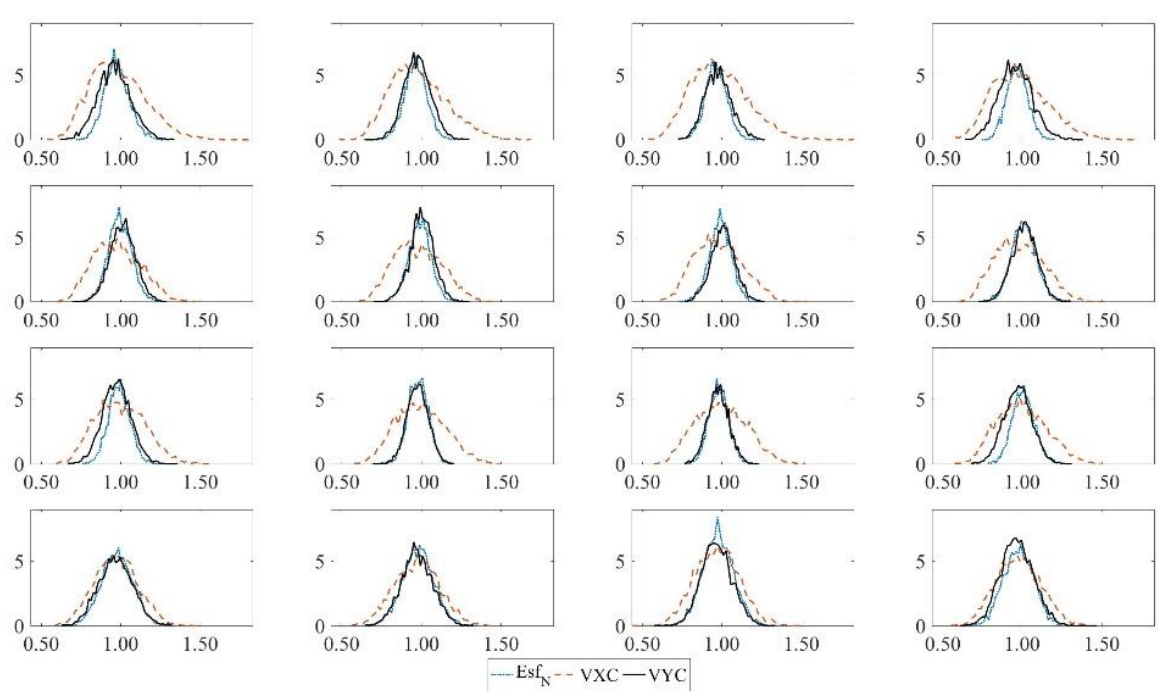

Figura 7. Histogramas de factores $A_{e a}$ en columnas - Marco X1 modelo M4C6

La figura 7 muestra los histogramas de $A_{e a}$ para las 16 columnas del marco X1 del modelo M4C6 para los parámetros de respuesta Esf $_{\mathrm{N}}$, VXC y VYC. Se observa que el valor máximo de $A_{e a}$ para el parámetro VXC es 1.50, mientras que para los parámetros VYC y Esf $f_{\mathrm{N}}$ es 1.25.

Las figuras 8 y 9 muestran los resultados para los parámetros de desplazamiento (Idmx e Idmy) del modelo M4C6. En dirección Y el valor máximo de $A_{e a}$ es 1.20 mientras que en dirección X es 1.40. La forma de los histogramas indica que no existe el mismo efecto en los desplazamientos de entrepiso en las dos direcciones (X y Y). Lo anterior sugiere en principio que el registro sísmico seleccionado afecta a los factores de amplificación por torsión accidental. 

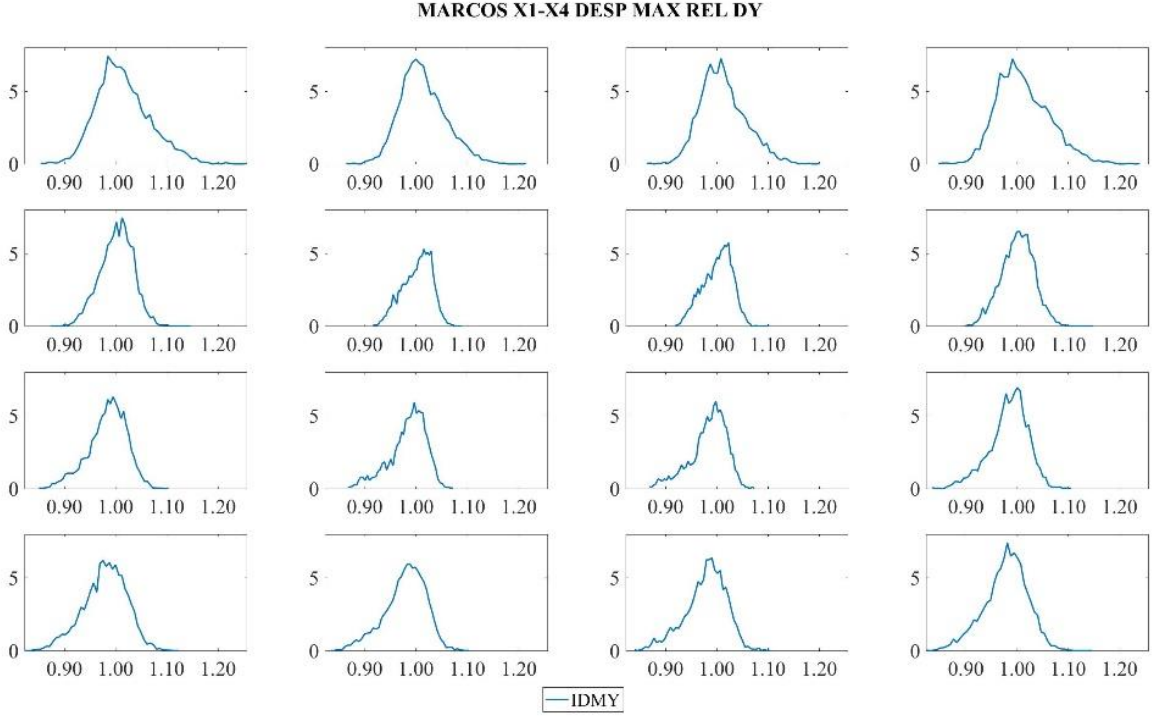

Figura 8. Histogramas de factores $A_{e a}$ parámetro de respuesta Idmy modelo M4C6


Figura 9. Histogramas de factores $A_{e a}$ parámetro de respuesta Idmx modelo M4C6

\section{Resultados de factores $A_{e a} \operatorname{con} p=0.02$ de modelos M4C6 y M4R6}

Para aplicar el procedimiento simplificado propuesto en esta investigación se utiliza un factor de amplificación por excentricidad accidental $A_{e a 2}$. Este factor tiene una probabilidad de excedencia aproximada de $2 \%$ ( $p \approx 0.02$ ). Antes de obtener este factor se seleccionaron factores $A_{e a}$ con probabilidad de excedencia igual a $2 \%(p=0.02)$ para cada parámetro de respuesta.

Como ejemplo, en esta sección se muestran sólo los factores $A_{e a}$ con $p=0.02$ del modelo M4C6. Para un mejor entendimiento de las gráficas que se muestran en las figuras 10 a 15 , se aclara lo siguiente: en la figura 10a se muestran los histogramas de factores $A_{e a}$ correspondientes a cada trabe del marco Y1 y se indica con una flecha la selección de los factores $A_{e a}$ con $p=0.02$ de las trabes entre los marcos X2 y 
X3. En la figura $10 \mathrm{~b}$ se grafican los factores $A_{e a}$ con $p=0.02$ de los tres parámetros de respuesta (VT, Mder y Mizq) para cada piso de las trabes entre los marcos X2 y X3.
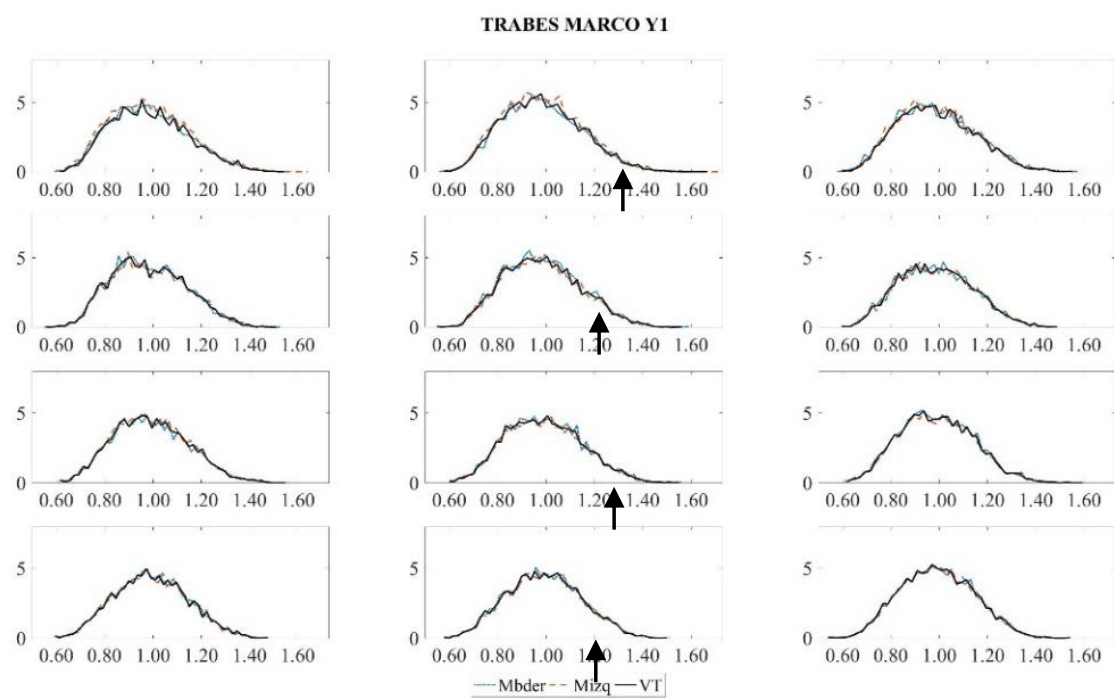

a)



Figura 10. a) Marco Y1 y b) selección de factores $A_{e a} \operatorname{con} p=0.02$ de trabes entre marcos X2 y X3 para los parámetros de respuesta VT, Mder y Mizq

En las figuras 11 a 14 se muestran los resultados de los factores $A_{e a}$ con $p=0.02$ del modelo M4C6. Por claridad, se omiten las leyendas en las gráficas, siendo el eje $\mathrm{X}$ el valor de los factores $A_{e a} \operatorname{con} p=0.02$ y el eje Y el número de piso del modelo.

Se colocaron etiquetas adicionales para indicar el marco del que se muestran resultados, así como las trabes correspondientes. La figura 11 muestra que para los marcos X, la mayoría de los valores de $A_{e a}$ oscilan entre 1.10 y 1.20. En la figura 12 se observa que para los marcos Y, la mayoría de los valores de $A_{\text {ea }}$ oscilan entre 1.20 a 1.40 .

Al igual que en los histogramas mostrados en la sección anterior, en las figuras 11 y 12 se observan valores distintos de los factores $A_{e a}$ con $p=0.02$ según la dirección de análisis. 



Figura 11. Factores $A_{e a}$ con $p=0.02$ - Trabes marcos X del modelo M4C6
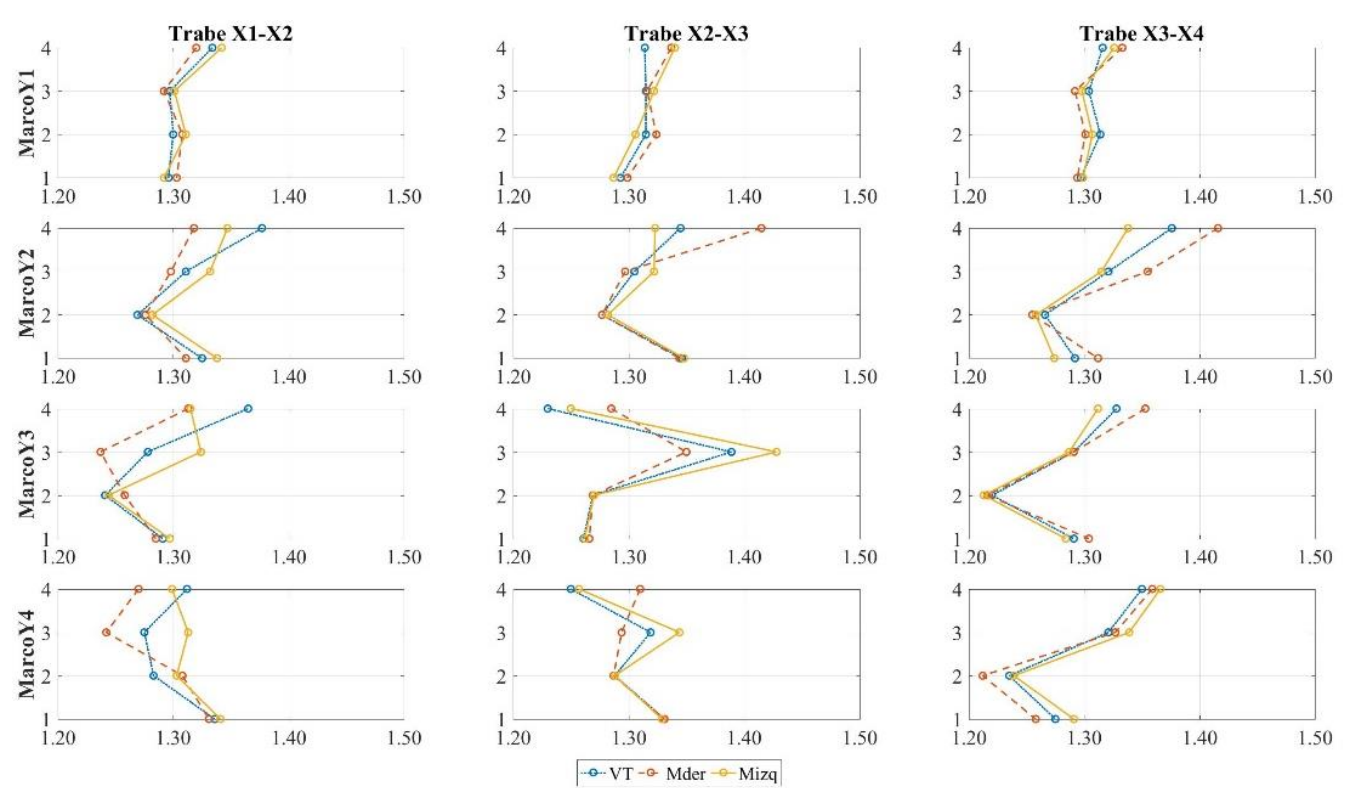

Figura 12. Factores $A_{e a} \operatorname{con} p=0.02$ - Trabes marcos Y modelo M4C6

La figura 13 muestra los resultados para columnas. Se observa que $A_{e a}$ con $p=0.02$ para VXC es del orden de 1.30 mientras que para VYC y Esf $_{\mathrm{N}}$ es de 1.15 a 1.20. 

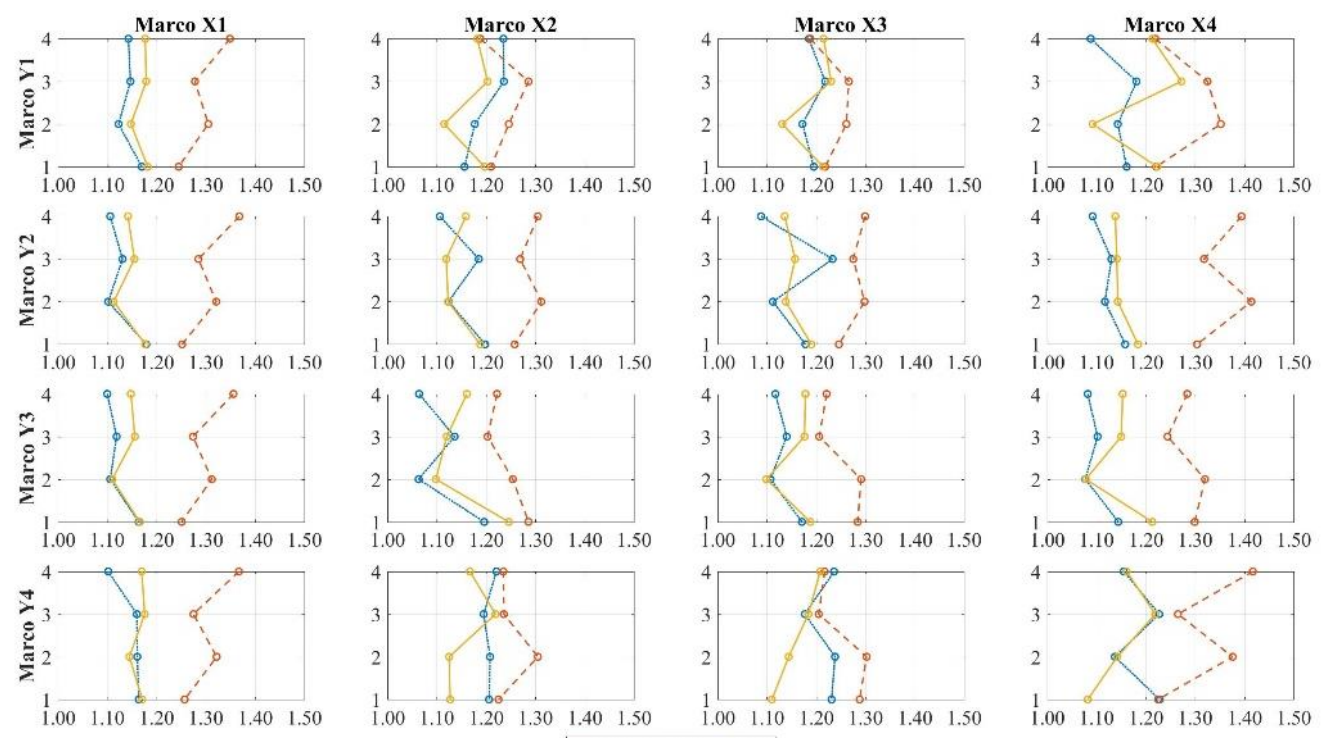

$$
-a-\text { Esf }_{\mathrm{N}}-\mathrm{a} \text {. } \mathrm{VXC}-\mathrm{a}-\mathrm{VYC}
$$

Figura 13. Factores $A_{e a}$ con $p=0.02$ - Columnas modelo M4C6

La figura 14 a y b muestra los resultados del modelo M4C6 de los parámetros de desplazamiento Idmx e Idmy con valores de $A_{e a}$ con $p=0.02$ que oscilan de 1.16 a 1.28 y de 1.02 a 1.14 respectivamente. La diferencia entre los resultados de Idmx e Idmy es aproximadamente del $12 \%$.

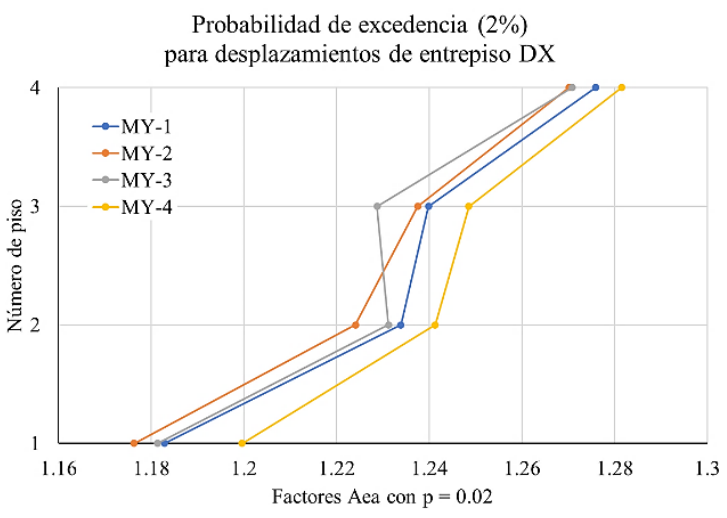

a)

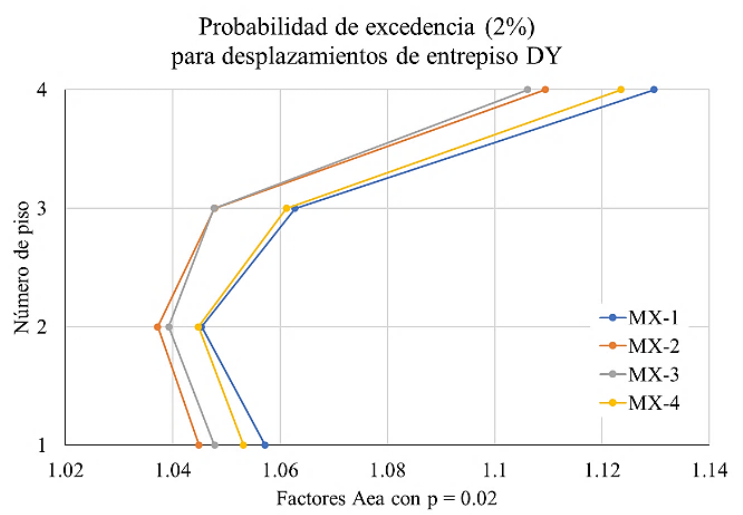

b)

Figura 14. Factores $A_{e a}$ con $p=0.02$ del parámetro Idmx e Idmy del modelo M4C6

De las figuras anteriores (11 a 14) se puede resumir lo siguiente: $\operatorname{los} A_{e a}$ con $p=0.02$ para trabes de los marcos $\mathrm{X}$ tienen variaciones desde 1.10 hasta 1.26, para cualquiera de los parámetros de respuesta. Para trabes de los marcos $\mathrm{Y}$, las variaciones son entre 1.21 y 1.43 , y en cuanto a los resultados para columnas las variaciones son entre 1.10 y 1.42. Los resultados para desplazamientos relativos máximos de entrepiso llevan a factores mayores en la dirección X que en la dirección Y.

\section{Resumen de factores $A_{e a} \operatorname{con} p=0.02$ de todos los modelos estudiados}

La sección anterior muestra los factores $A_{e a}$ con $p=0.02$ del modelo M4C6. Para incluir los resultados de todos los modelos se calcularon valores estadísticos como: la media, la desviación estándar, el coeficiente de variación y la varianza. Adicionalmente, se reportan los valores mínimos y máximos de 
dichos factores. Lo resultados para todos los parámetros de respuesta seleccionados se muestran en las tablas 6 a 13. La nomenclatura utilizada en las tablas 6 a 13 es la siguiente:

$A_{e a}$ min. - Corresponde a $A_{e a}$ mínimo.

$A_{e a}$ máx. - Corresponde a $A_{e a}$ máximo.

$A_{e a}$ media - Corresponde a $A_{e a}$ promedio o media geométrica de los datos.

$A_{e a}$ media* - Corresponde al mismo valor anterior pero redondeado a 2 decimales.

$A_{e a}$ Desv. Std - Corresponde a la desviación estándar de $A_{e a}$.

$A_{e a}$ Varianza - Corresponde a la varianza de $A_{e a}$.

$A_{e a}$ Coef. Var. - Corresponde al coeficiente de variación de $A_{e a}$.

Para la interpretación de los resultados reportados en las tablas siguientes se aclara que:

Parámetros trabes MX y MY: Incluye los resultados de los factores $A_{e a}$ con $p=0.02$ de los parámetros estudiados en trabes (Mder, Mizq y VT) para todos los marcos en las direcciones X y Y respectivamente.

Parámetros columnas: Incluye los resultados de los factores $A_{e a}$ con $p=0.02$ de los parámetros estudiados en columnas $\left(\mathrm{Esf}_{\mathrm{N}}, \mathrm{VXC}\right.$ y VYC).

Parámetros Idmx e Idmy: Incluye los resultados de los factores $A_{e a}$ con $p=0.02$ de los parámetros Idmx e Idmy respectivamente de todos los pisos del modelo.

Tabla 6. Resumen valores estadísticos de $\operatorname{los} A_{e a}$ con $\mathrm{p}=0.02$ (Modelo M4C6)

\begin{tabular}{|c|c|c|c|c|c|}
\hline $\begin{array}{l}\text { Valores de factores } \\
\mathrm{A}_{\mathrm{ea}} \text { con } \mathrm{p}=0.02\end{array}$ & $\begin{array}{l}\text { Parámetros } \\
\text { trabes MX }\end{array}$ & $\begin{array}{l}\text { Parámetros } \\
\text { trabes MY }\end{array}$ & $\begin{array}{l}\text { Parámetros } \\
\text { columnas }\end{array}$ & $\begin{array}{l}\text { Parámetro } \\
\text { Idmx }\end{array}$ & $\begin{array}{l}\text { Parámetro } \\
\text { Idmy }\end{array}$ \\
\hline $\mathrm{A}_{\mathrm{ea}} \min$. & 1.091 & 1.212 & 1.063 & 1.014 & 1.168 \\
\hline Aea máx. & 1.264 & 1.428 & 1.417 & 1.133 & 1.282 \\
\hline Aea media & 1.168 & 1.304 & 1.199 & 1.060 & 1.206 \\
\hline Aea media* & 1.200 & 1.300 & 1.200 & 1.100 & 1.200 \\
\hline Aea Desv. Std. & 0.0372 & 0.0384 & 0.0745 & 0.0395 & 0.0405 \\
\hline Aea Varianza & 0.0014 & 0.0015 & 0.0056 & 0.0016 & 0.0016 \\
\hline A ea Coef. Var. & $3.10 \%$ & $2.96 \%$ & $6.21 \%$ & $3.59 \%$ & $3.38 \%$ \\
\hline
\end{tabular}

Tabla 7. Resumen de valores estadísticos de $\operatorname{los} A_{e a}$ con $\mathrm{p}=0.02$ (Modelo M4R6)

\begin{tabular}{llllll}
\hline $\begin{array}{l}\text { Valores de factores } \\
\mathrm{A}_{\text {ea }} \text { con } \mathrm{p}=0.02\end{array}$ & $\begin{array}{l}\text { Parámetros } \\
\text { trabes MX }\end{array}$ & $\begin{array}{l}\text { Parámetros } \\
\text { trabes MY }\end{array}$ & $\begin{array}{l}\text { Parámetros } \\
\text { columnas }\end{array}$ & $\begin{array}{l}\text { Parámetro } \\
\text { Idmx }\end{array}$ & $\begin{array}{l}\text { Parámetro } \\
\text { Idmy }\end{array}$ \\
\hline $\mathrm{A}_{\text {ea }}$ min. & 1.102 & 1.201 & 1.059 & 0.978 & 1.238 \\
$\mathrm{~A}_{\text {ea }}$ máx. & 1.279 & 1.483 & 1.577 & 1.138 & 1.325 \\
$\mathrm{~A}_{\text {ea }}$ media & 1.151 & 1.330 & 1.207 & 1.035 & 1.279 \\
$\mathrm{~A}_{\text {ea }}$ media* & 1.200 & 1.300 & 1.200 & 1.050 & 1.300 \\
$\mathrm{~A}_{\text {ea }}$ Desv. Std. & 0.0295 & 0.0437 & 0.1077 & 0.0470 & 0.0286 \\
$\mathrm{~A}_{\text {ea }}$ Varianza & 0.0009 & 0.0019 & 0.0116 & 0.0022 & 0.0008 \\
$\mathrm{~A}_{\text {ea }}$ Coef. Var. & $2.45 \%$ & $3.36 \%$ & $8.97 \%$ & $4.70 \%$ & $2.20 \%$ \\
\hline
\end{tabular}

Tabla 8. Resumen de valores estadísticos de los $A_{e a}$ con $\mathrm{p}=0.02$ (Modelo M7C6)

\begin{tabular}{|c|c|c|c|c|c|}
\hline $\begin{array}{l}\text { Valores de factores } \\
A_{\text {ea }} \text { con } p=0.02\end{array}$ & $\begin{array}{l}\text { Parámetros } \\
\text { trabes MX }\end{array}$ & $\begin{array}{l}\text { Parámetros } \\
\text { trabes MY }\end{array}$ & $\begin{array}{l}\text { Parámetros } \\
\text { columnas }\end{array}$ & $\begin{array}{l}\text { Parámetro } \\
\text { Idmx }\end{array}$ & $\begin{array}{l}\text { Parámetro } \\
\text { Idmy }\end{array}$ \\
\hline Aea min. & 1.101 & 1.111 & 1.071 & 1.034 & 1.024 \\
\hline Aea máx. & 1.351 & 1.345 & 1.514 & 1.187 & 1.233 \\
\hline Aea media & 1.189 & 1.241 & 1.197 & 1.106 & 1.132 \\
\hline Aea media* & 1.200 & 1.200 & 1.200 & 1.100 & 1.100 \\
\hline Aea Desv. Std. & 0.0411 & 0.0459 & 0.0643 & 0.0464 & 0.0649 \\
\hline A ea Varianza & 0.0017 & 0.0021 & 0.0041 & 0.0021 & 0.0042 \\
\hline $\mathrm{A}_{\mathrm{ea}}$ Coef. Var. & $3.43 \%$ & $3.83 \%$ & $5.36 \%$ & $4.21 \%$ & $5.90 \%$ \\
\hline
\end{tabular}


Tabla 9. Resumen de valores estadísticos de $\operatorname{los} A_{e a}$ con $\mathrm{p}=0.02$ (Modelo M7R6)

\begin{tabular}{llllll}
\hline $\begin{array}{l}\text { Valores de factores } \\
\mathrm{A}_{\text {ea }} \text { con } \mathrm{p}=0.02\end{array}$ & $\begin{array}{l}\text { Parámetros } \\
\text { trabes MX }\end{array}$ & $\begin{array}{l}\text { Parámetros } \\
\text { trabes MY }\end{array}$ & $\begin{array}{l}\text { Parámetros } \\
\text { columnas }\end{array}$ & $\begin{array}{l}\text { Parámetro } \\
\text { Idmx }\end{array}$ & $\begin{array}{l}\text { Parámetro } \\
\text { Idmy }\end{array}$ \\
\hline $\mathrm{A}_{\text {ea }}$ min. & 1.122 & 1.088 & 1.054 & 1.051 & 1.094 \\
$\mathrm{~A}_{\text {ea }}$ máx. & 1.264 & 1.301 & 1.437 & 1.169 & 1.208 \\
$\mathrm{~A}_{\text {ea }}$ media & 1.194 & 1.189 & 1.188 & 1.114 & 1.133 \\
$\mathrm{~A}_{\text {ea }}$ media* & 1.200 & 1.200 & 1.200 & 1.100 & 1.100 \\
$\mathrm{~A}_{\text {ea }}$ Desv. Std. & 0.0290 & 0.0357 & 0.0654 & 0.0378 & 0.0311 \\
$\mathrm{~A}_{\text {ea Varianza }}$ & 0.0008 & 0.0013 & 0.0043 & 0.0014 & 0.0010 \\
$\mathrm{~A}_{\text {ea }}$ Coef. Var. & $2.42 \%$ & $2.98 \%$ & $5.45 \%$ & $3.44 \%$ & $2.83 \%$ \\
\hline
\end{tabular}

Tabla 10. Resumen de valores estadísticos de los $A_{e a \text { a }} \operatorname{con} \mathrm{p}=0.02$ (Modelo M7C8)

\begin{tabular}{llllll}
\hline Valores de factores & $\begin{array}{l}\text { Parámetros } \\
\text { trabes MX }\end{array}$ & $\begin{array}{l}\text { Parámetros } \\
\text { trabes MY }\end{array}$ & $\begin{array}{l}\text { Parámetros } \\
\text { columnas }\end{array}$ & $\begin{array}{l}\text { Parámetro } \\
\text { Idmx }\end{array}$ & $\begin{array}{l}\text { Parámetro } \\
\text { Idmy }\end{array}$ \\
\hline Aea min. & 1.078 & 1.062 & 1.032 & 0.993 & 1.010 \\
Aea máx. & 1.233 & 1.231 & 1.307 & 1.082 & 1.094 \\
$A_{\text {ea }}$ media & 1.163 & 1.165 & 1.143 & 1.039 & 1.056 \\
A ea media* $_{\text {Aea Desv. Std. }}$ & 1.200 & 1.200 & 1.100 & 1.050 & 1.100 \\
A ea Varianza $_{A_{\text {ea }} \text { Coef. Var. }}^{0.0303}$ & 0.0009 & 0.0316 & 0.0409 & 0.0257 & 0.0207 \\
\hline
\end{tabular}

Tabla 11. Resumen de valores estadísticos de los $\mathrm{A}_{\mathrm{ea}}$ con $\mathrm{p}=0.02$ (Modelo M7R8)

\begin{tabular}{|c|c|c|c|c|c|}
\hline $\begin{array}{l}\text { Valores de factores } \\
A_{\text {ea }} \text { con } \mathrm{p}=0.02\end{array}$ & $\begin{array}{l}\text { Parámetros } \\
\text { trabes MX }\end{array}$ & $\begin{array}{l}\text { Parámetros } \\
\text { trabes MY }\end{array}$ & $\begin{array}{l}\text { Parámetros } \\
\text { columnas }\end{array}$ & $\begin{array}{l}\text { Parámetro } \\
\text { Idmx }\end{array}$ & $\begin{array}{l}\text { Parámetro } \\
\text { Idmy }\end{array}$ \\
\hline$A_{\text {ea }} \min$. & 1.102 & 1.106 & 1.057 & 1.008 & 1.034 \\
\hline A ea máx. & 1.295 & 1.301 & 1.336 & 1.106 & 1.169 \\
\hline$A_{\text {ea }}$ media & 1.173 & 1.184 & 1.153 & 1.054 & 1.093 \\
\hline Aea media* & 1.200 & 1.200 & 1.200 & 1.100 & 1.100 \\
\hline Aea Desv. Std. & 0.0339 & 0.0332 & 0.0459 & 0.0274 & 0.0405 \\
\hline Aea Varianza & 0.0012 & 0.0011 & 0.0021 & 0.0008 & 0.0016 \\
\hline$A_{\text {ea }}$ Coef. Var. & $2.83 \%$ & $2.77 \%$ & $3.82 \%$ & $2.49 \%$ & $3.68 \%$ \\
\hline
\end{tabular}

Tabla 12. Resumen valores estadísticos de los $A_{e a} \operatorname{con} \mathrm{p}=0.02$ (Modelo M10C8)

\begin{tabular}{|c|c|c|c|c|c|}
\hline $\begin{array}{l}\text { Valores de factores } \\
A_{\text {ea }} \text { con } \mathrm{p}=0.02\end{array}$ & $\begin{array}{l}\text { Parámetros } \\
\text { trabes MX }\end{array}$ & $\begin{array}{l}\text { Parámetros } \\
\text { trabes MY }\end{array}$ & $\begin{array}{l}\text { Parámetros } \\
\text { columnas }\end{array}$ & $\begin{array}{l}\text { Parámetro } \\
\text { Idmx }\end{array}$ & $\begin{array}{l}\text { Parámetro } \\
\text { Idmy }\end{array}$ \\
\hline $\mathrm{A}_{\mathrm{ea}} \min$. & 1.025 & 1.057 & 1.032 & 0.989 & 0.990 \\
\hline A ea máx. & 1.296 & 1.363 & 1.341 & 1.104 & 1.060 \\
\hline A ea media & 1.153 & 1.171 & 1.129 & 1.029 & 1.017 \\
\hline A ea media* & 1.200 & 1.200 & 1.100 & 1.050 & 1.000 \\
\hline A ea Desv. Std. & 0.0497 & 0.0458 & 0.0532 & 0.0292 & 0.0177 \\
\hline A ea Varianza & 0.0025 & 0.0021 & 0.0028 & 0.0009 & 0.0003 \\
\hline $\mathrm{A}_{\mathrm{ea}}$ Coef. Var. & $4.14 \%$ & $3.82 \%$ & $4.84 \%$ & $2.92 \%$ & $1.77 \%$ \\
\hline
\end{tabular}

Tabla 13. Resumen de valores estadísticos de los $A_{e a} \operatorname{con} \mathrm{p}=0.02$ (Modelo M10R8)

\begin{tabular}{llllll}
\hline $\begin{array}{l}\text { Valores de factores } \\
\mathrm{A}_{\text {ea }} \text { con } \mathrm{p}=0.02\end{array}$ & $\begin{array}{l}\text { Parámetros } \\
\text { trabes MX }\end{array}$ & $\begin{array}{l}\text { Parámetros } \\
\text { trabes MY }\end{array}$ & $\begin{array}{l}\text { Parámetros } \\
\text { columnas }\end{array}$ & $\begin{array}{l}\text { Parámetro } \\
\text { Idmx }\end{array}$ & $\begin{array}{l}\text { Parámetro } \\
\text { Idmy }\end{array}$ \\
\hline $\mathrm{A}_{\text {ea }}$ min. & 1.043 & 1.088 & 1.063 & 1.021 & 1.016 \\
$\mathrm{~A}_{\text {ea }}$ máx. & 1.274 & 1.276 & 1.326 & 1.191 & 1.076 \\
$A_{\text {ea media }}$ & 1.175 & 1.177 & 1.154 & 1.070 & 1.041 \\
Aea media* $_{\text {Aea Desv. Std. }}$ & 1.200 & 1.200 & 1.200 & 1.100 & 1.050 \\
$A_{\text {ea Varianza }}$ & 0.0377 & 0.0263 & 0.0457 & 0.0413 & 0.0171 \\
$\mathrm{~A}_{\mathrm{ea}}$ Coef. Var. & 0.0014 & 0.0007 & 0.0021 & 0.0017 & 0.0003 \\
\hline
\end{tabular}

En las tablas 6 a 13 se observa que los valores de $A_{e a}$ media* oscilan entre 1.10 y 1.30 para los parámetros de trabes y de columnas. Para los parámetros Idmx e Idmy $\mathrm{A}_{\text {ea }}$ media* oscilan entre 1.05 y 1.10. 
Los resultados de las tablas 6 a 13 muestran que el coeficiente de variación máximo es de $8.97 \%$ correspondiente al modelo M4R6. Debido a que este valor es menor al 10\% se puede considerar que se tiene una precisión buena de los datos obtenidos de acuerdo con DANE (2008). Además, en ningún caso la varianza se excede de $1 \%$, indicando que la media es representativa de los datos obtenidos y que no existen grandes cambios entre los mismos.

Por otra parte, los valores máximos de $A_{e a}$ con $p=0.02$, se reducen conforme aumenta la altura de los modelos. Lo anterior, se ilustra en la figura 15 la cual muestra el parámetro Idmy del modelo M10R8. La figura 15, comparada con la figura 14 indica que se requieren factores $A_{e a}$ con $p=0.02$ mayores en modelos de baja altura (menos de 7 pisos).

Probabilidad de excedencia (2\%) para desplazamientos de entrepiso DY

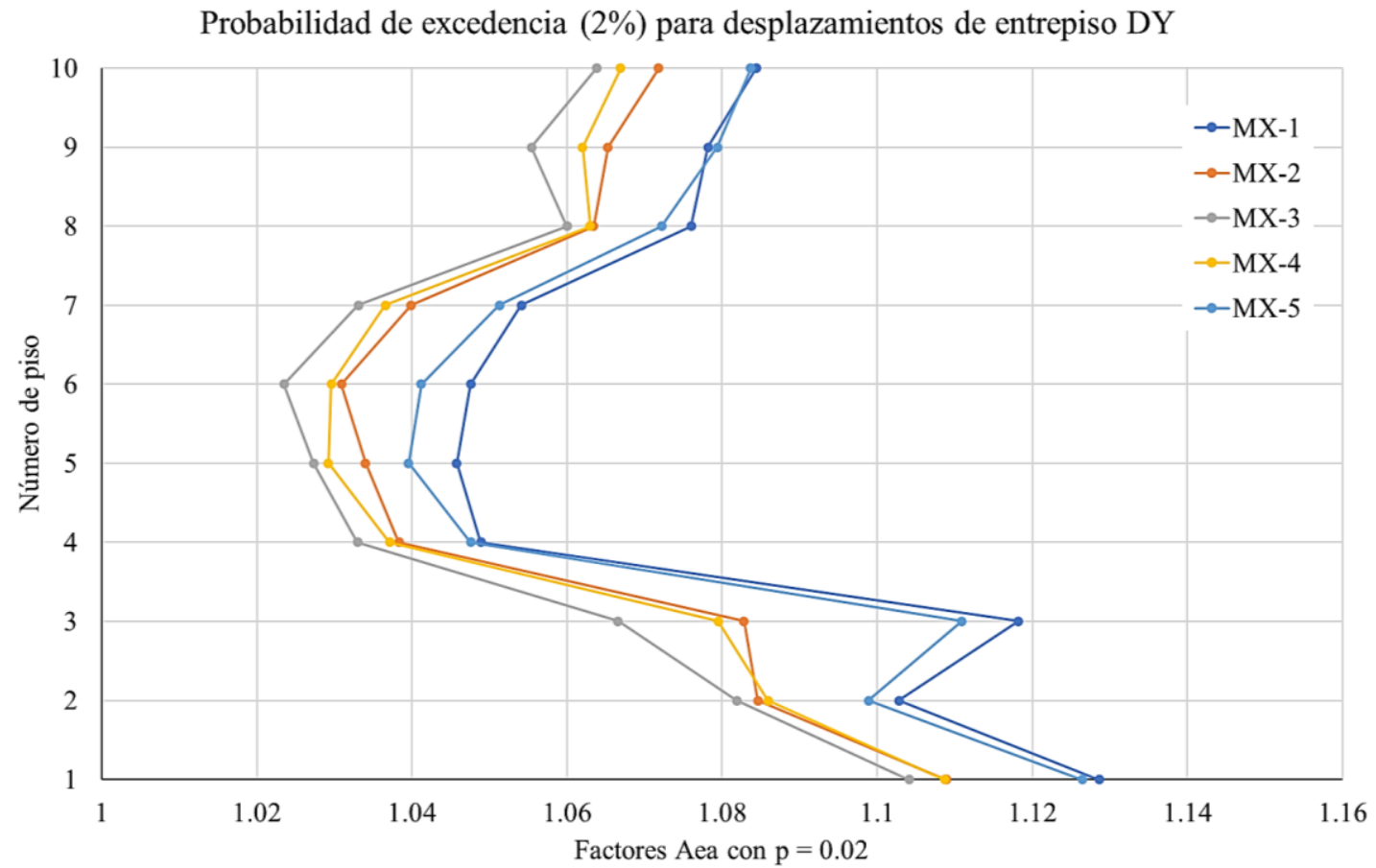

Figura 15. Factores $A_{e a}$ con $p=0.02$ - Parámetro Idmy del modelo M10R8

En rigor parece necesario aplicar factores distintos de $A_{e a}$ con $p=0.02$ para cada modelo, cada elemento estructural y cada piso. Debido a lo anterior, se calcularon algunos parámetros estadísticos de $A_{e a}$ con $p=0.02$ para cada modelo y verificar que estadísticamente un valor promedio es representativo de todos los datos. Con el fin de aplicar dicho valor al procedimiento simplificado propuesto. De lo anterior, se obtuvieron valores que oscilan entre 1.05 y 1.30, sin embargo, la aplicación de estos valores no cubre a todos los de modelos, por lo que se realizó otro tratamiento estadístico similar al descrito con anterioridad a los datos de todos modelos, diferenciando los datos de los modelos cuadrados de los datos de los modelos rectangulares. Las tablas 14 y 15 muestran los resultados mencionados respectivamente y utilizan la misma nomenclatura que las tablas 6 a 13.

La tabla 14 corresponde a todos los modelos cuadrados y muestra $A_{e a}$ media* igual a 1.20 para los parámetros de trabes y columnas. Por otro lado, para los parámetros cinemáticos IDMX e IDMY se obtiene $A_{\text {ea }}$ media* igual a 1.10 . 
Tabla 14. Resumen de factores $A_{e a}$ con $\mathrm{p}=0.02$ modelos cuadrados

\begin{tabular}{llllll}
\hline Valores de factores & $\begin{array}{l}\text { Parámetros } \\
\text { trabes MX }\end{array}$ & $\begin{array}{l}\text { Parámetros } \\
\text { trabes MY }\end{array}$ & $\begin{array}{l}\text { Parámetros } \\
\text { columnas }\end{array}$ & $\begin{array}{l}\text { Parámetro } \\
\text { Idmx }\end{array}$ & $\begin{array}{l}\text { Parámetro } \\
\text { Idmy }\end{array}$ \\
\hline$A_{\text {ea }}$ min. $p=0.02$ & 1.025 & 1.057 & 1.032 & 0.989 & 0.990 \\
$A_{\text {ea }}$ máx. & 1.351 & 1.428 & 1.514 & 1.187 & 1.282 \\
$A_{\text {ea }}$ media & 1.167 & 1.206 & 1.160 & 1.055 & 1.083 \\
$A_{\text {ea }}$ media* & 1.200 & 1.200 & 1.200 & 1.100 & 1.100 \\
$A_{\text {ea }}$ Desv. Std. & 0.0439 & 0.0653 & 0.0650 & 0.0467 & 0.0777 \\
$A_{\text {ea Varianza }}$ & 0.0019 & 0.0043 & 0.0042 & 0.0022 & 0.0060 \\
$A_{\text {ea }}$ Coef. Var. & $3.66 \%$ & $5.44 \%$ & $5.42 \%$ & $4.24 \%$ & $7.06 \%$ \\
\hline
\end{tabular}

La tabla 15 corresponde a los datos de todos los modelos rectangulares y muestra $A_{e a}$ media* igual a 1.20 para los parámetros de trabes y columnas. Por otro lado, para los parámetros cinemáticos Idmx e Idmy se obtiene $A_{e a}$ media* igual a 1.10 . Notar que el uso de las medias anteriores lleva a diferencias del $24 \%$ con respecto a los valores $A_{e a}$ máximos.

Tabla 15. Resumen de factores $A_{e a}$ con $\mathrm{p}=0.02$ modelos rectangulares

\begin{tabular}{llllll}
\hline $\begin{array}{l}\text { Valores de factores } \\
\mathrm{A}_{\text {ea }} \text { con } p=0.02\end{array}$ & $\begin{array}{l}\text { Parámetros } \\
\text { trabes MX }\end{array}$ & $\begin{array}{l}\text { Parámetros } \\
\text { trabes MY }\end{array}$ & $\begin{array}{l}\text { Parámetros } \\
\text { columnas }\end{array}$ & $\begin{array}{l}\text { Parámetro } \\
\text { Idmx }\end{array}$ & $\begin{array}{l}\text { Parámetro } \\
\text { Idmy }\end{array}$ \\
\hline A $_{\text {ea }}$ min. & 1.043 & 1.088 & 1.054 & 0.978 & 1.016 \\
A ea máx. $_{\text {Aea media }}^{1.295}$ & 1.483 & 1.577 & 1.191 & 1.325 \\
Aea media* $_{\text {Aea Desv. Std. }}$ & 1.176 & 1.204 & 1.170 & 1.072 & 1.111 \\
Aea Varianza $_{\text {A ea Coef. Var. }}^{1.200}$ & 1.200 & 1.200 & 1.100 & 1.100 \\
\hline
\end{tabular}

De las tablas 14 y 15 se observa que $A_{e a}$ máximo es de 1.514 para modelos cuadrados y de 1.577 para modelos rectangulares. En ambos casos el valor corresponde a alguno de los parámetros de columnas. Lo anterior, indica que los elementos estructurales más afectados por la excentricidad accidental son las columnas. El coeficiente de variación máximo tiene un valor de $7.06 \%$ para los modelos cuadrados y de $7.52 \%$ para los modelos rectangulares. Dados estos valores menores que $10 \%, \mathrm{~A}_{\text {ea }}$ media* igual a 1.20, es estadísticamente representativa de todos los datos. El resultado anterior se mantiene para los modelos rectangulares y para los modelos cuadrados sin importar la dirección de análisis. Por lo tanto, estadísticamente, a partir de los datos anteriores se puede decir, que no hay diferencias importantes debidas al registro sísmico utilizado. Considerando los valores máximos de $A_{e a}$ listados en las tablas 14 y 15 , en la tabla 16 se muestra la probabilidad de exceder dichos máximos y se comparan contra la probabilidad de exceder el valor de $A_{e a}$ media* $=1.20$. Como se puede observar, para los valores máximos en ningún caso se tienen probabilidades de excedencia mayores al $0.02 \%$. Por otro lado, para $A_{e a}$ media* las probabilidades de excederla en ningún caso son mayores de $2 \%$.

Tabla 16. Probabilidades de excedencia de los factores $A_{e a}$ máx. y $A_{e a}$ media*.

\begin{tabular}{|l|c|c|c|c|}
\hline Parámetros de: & $\boldsymbol{p}$ de $\boldsymbol{A}_{\boldsymbol{e a}}$ máx. = 1.514 & $\boldsymbol{p}$ de $\boldsymbol{A}_{\boldsymbol{e a}}=\mathbf{1 . 2 0}$ & $\boldsymbol{p}$ de $\boldsymbol{A}_{\boldsymbol{e a}}$ máx. = 1.577 & $\boldsymbol{p}$ de $\boldsymbol{A}_{\boldsymbol{e a}}=\mathbf{1 . 2 0}$ \\
\hline Trabes & $0.0020 \%$ & $1.9425 \%$ & $0.0106 \%$ & $1.0427 \%$ \\
\hline Columnas & $0.0049 \%$ & $1.1102 \%$ & $0.0067 \%$ & $0.9746 \%$ \\
\hline IDMX & $0.0000 \%$ & $0.0332 \%$ & $0.0000 \%$ & $0.0167 \%$ \\
\hline IDMY & $0.0002 \%$ & $0.7940 \%$ & $0.0050 \%$ & $0.7930 \%$ \\
\hline
\end{tabular}




\section{CONCLUSIONES}

El objetivo principal de esta investigación fue establecer un procedimiento simplificado para tomar en cuenta los efectos de la excentricidad accidental en el diseño sísmico de edificios. El procedimiento propuesto permite calcular los elementos mecánicos y cinemáticos requeridos por el diseñador cuando se tiene un edificio con excentricidad accidental y que al mismo tiempo esté sujeto a un sismo. Los resultados se limitan a modelos con excentricidad natural $e_{s}=0$ y a análisis lineales; sin embargo, se pretende abordar en futuros trabajos el comportamiento no lineal de los edificios. El estudio considera edificios para oficinas estructurados con marcos de concreto reforzado. El procedimiento de diseño se enfoca a edificios con distribuciones uniformes de masa y rigidez, tanto en planta como en elevación. Asimismo, se anticipa que su aplicación debiera limitarse a edificios que por lo menos tengan tres marcos en cada dirección ortogonal.

El procedimiento simplificado propuesto usa una relación simple que estima elementos mecánicos y cinemáticos para diseño a partir de la amplificación de los correspondientes valores obtenidos de un análisis del correspondiente modelo torsionalmente balanceado.

A partir de los resultados de las tablas 14 y 15, se recomienda utilizar un factor de amplificación por excentricidad accidental con $p \approx 2 \%\left(\mathrm{~A}_{\mathrm{ea} 2}\right)$ igual a 1.20 . Este factor es aplicable a cualquier elemento mecánico o cinemático obtenido de modelos a base de marcos de concreto reforzado. Aun cuando el estudio se realizó para el caso extremo de sistemas con $e_{s}=0$, su aplicación no se limita, en principio, a sistemas con $e_{s} \neq 0$. En este último caso la amplificación por torsión accidental se aplicaría a los resultados del análisis del modelo con su excentricidad natural $\left(e_{s} \neq 0\right)$ y $e_{a}=0$.

Mediante un valor $A_{\text {ea } 2}=1.20$ se tienen coeficientes de variación máximos de $7.52 \%$ menores que $10 \%$ por lo que se puede afirmar que se obtuvo una precisión buena de acuerdo con DANE (2008). En ningún caso se excede un valor del $1 \%$ para la varianza, indicando que la media de los datos igual a 1.20 es representativa de todos los datos obtenidos y que no existen grandes cambios entre los mismos.

El uso de factores de $A_{e a}$ mayores a 1.20 conlleva a tener probabilidades de excedencia de los elementos mecánicos y cinemáticos menores del $0.02 \%$. Lo anterior se resumió en la tabla 16.

Se corroboró que la probabilidad de exceder un determinado factor $A_{e a}$ de cualquier parámetro de respuesta en modelos con $e_{a} \neq 0$ es menor en entrepisos inferiores que en los superiores.

Los resultados muestran que en el $60 \%$ de los casos, los factores estimados de $A_{e a}$ con $p=0.02$ obtenidos en modelos rectangulares son mayores que en los modelos cuadrados.

Se observa que para modelos de baja altura (menos de 7 pisos) se requieren factores $A_{e a}$ con $p=0.02$ aproximadamente $20 \%$ mayores que los requeridos en modelos de mediana altura (7-10 pisos).

Aunque el factor $A_{e a 2}=1.20$ es representativo en general de la amplificación de los parámetros de respuesta con posibilidad de experimentar torsión accidental, se observó que pueden seleccionarse valores más específicos dependiendo del elemento estructural, su ubicación en el modelo y del parámetro de respuesta.

En resumen, se realizó un estudio amplio del efecto de la torsión accidental en varios parámetros de la respuesta estructural que inciden en el diseño sísmico de edificios. Este estudio permitió establecer un procedimiento simplificado para incorporar los efectos de la torsión accidental en el diseño sísmico de edificios. El procedimiento estima los elementos mecánicos y cinemáticos con una probabilidad de excedencia aproximada del $2 \%$, a partir de una relación simple que requiere un único análisis del modelo con $e_{a}=0$ y la amplificación de sus respuestas por un factor $A_{\text {ea } 2}=1.20$. 


\section{REFERENCIAS}

ASCE (2010). American Society of Civil Engineers Minimum design loads for buildings and other structures-ASCE. Standard 7-05: ASCE. EUA.

Associate Committee on the National Building Code (ACNBC). (1980). "National building code of Canada" (NBCC), National Research Council of Canada, Ottawa.

DANE. (2008). Estimación e interpretación del coeficiente de variación de la encuesta concensal. Colombia: Departamento Administrativo Nacional de Estadística.

De la Colina, J., y Benítez, B., (2006). Estudio probabilista sobre excentricidad accidental en edificios utilizando el método Monte Carlo. Puerto Vallarta, Jalisco: Sociedad Mexicana de Ingeniería Estructural, XV Congreso Nacional de Ingeniería Estructural.

De la Colina, J., González, C. y Valdés, J., (2016). Accidental eccentricities, frame shear forces and ductility demands of buildings with uncertainties of stiffness and live load: Engineering Structures 124, 113127. DOI: $10.1016 /$ j.engstruct.2016.06.012

De la Colina, J., Ruiz, S. y Benítez, B., (2011). Accidental eccentricity of story shear for low-rise office buildings: Journal of Structural Division, ASCE, 137 (4), 513- 520. DOI: 10.1061/(ASCE)ST.1943541X.0000302

De la Llera, J. y Chopra, A., (1994). Accidental and natural torsion in earthquake response and design of buildings: Report No. UCB/EERC-94/07. Earthquake Engineering Research Center. University of California at Berkeley.

Díaz E., (1995). Selección del número de replicaciones en un estudio de simulación. Bilbao España: Departamento de econometría y estadística, Universidad del País Vasco.

ECS, (2004). ECS. Eurocode 8: design of structures for earthquake resistance. Part 1: general rules, seismic actions and rules for buildings. EN 1998-1:2004. Brussels Belgium: European Committee for Standardization.

Escobar S., A., Mendoza S., A. y Gómez M., R., (2002). "Procedimiento simplificado para diseño por torsión sísmica estática". XIII Congreso Nacional de Ingeniería Estructural.

Escobar S., A., Mendoza S., A. y Gómez M., R., (2004). Diseño simplificado para diseño por torsión sísmica estática. Revista de Ingeniería Sísmica, Issue 70, pp. 77-107. DOI: https://doi.org/10.18867/ris.70.52

García D. V., (2015). Análisis de las probabilidades de excedencia de las respuestas de modelos estructurales con torsión sujetos a sismos usando simulación Monte Carlo. Toluca, Méx.: Tesis de Maestría UAEMex.

Hernández J., and López O. (2004). "Dependence of accidental torsion on structural system properties". 13th World Conference on Earthquake Engineering, Vancouver, B.C., Canada. Paper No. 1165.

Hogg, S. y Craig, F., (1995). Introduction to mathematical statics. 5th ed. New Jersey: Prentice-Hall: s.n.

ICC, (2009). ICC. International building code. International Code Council: Inc.

Kang L. N., (1996). Canny-E User's Manual, Three-dimension nonlinear dynamic structural analysis computer program package: Canny Consultants Pte. Ldt., Singapur.

Metropolis, N; Ulam, S., (1949). The Monte Carlo method. Journal of the American Statistical Association, 44, 335-341. DOI:10.2307/2280232 https://www.jstor.org/stable/2280232

Nelson H. (2001). Influencia de la torsión accidental en el diseño sísmico de edificios. Tesis. Universidad nacional de ingeniería. Lima Perú.

Padilla, A., (2002). Evaluación de un requisito complementario a los criterios de diseño por torsión del RCDF en sus edificios 1995 y 2001: Tesis maestría, DEPFI, UNAM.

RC CdMx-2017, (2017). Reglamento de Construcciones para la Ciudad de México (RCDF) "Normas técnicas complementarias para diseño por sismo".

Soriano, A. y Ruiz, S., (2001). Cargas vivas máximas para edificios de oficinas en la Ciudad de México: Reporte de investigación, Instituto de ingeniería UNAM. 
Estimación de los efectos de torsión accidental sísmica a partir de la amplificación de la respuesta de un modelo torsionalmente balanceado

Zárate, G., Ayala, A. G. y García, O., (2003). Método sísmico estático para edificios asimétricos: revisión de enfoques. Distrito Federal, Méx.: Revista de Ingeniería Sísmica, núm. 69, julio-diciembre, pp. 2544. DOI:10.18867/ris.69.217 